\title{
Ultrasound Guided Wire Localization of Anal Tract in Imperforate
}

\section{Anus}

\author{
Seyed Ali Alamdaran, ${ }^{1}$ Jahanbakhsh Hashemi, ${ }^{2}$ Ali Feyzi, ${ }^{2}$ Ali Azadmand, ${ }^{3}$ Masoud Mahdavi Rashed, ${ }^{2}$ \\ Samira Jafari, ${ }^{2}$ and Marjan Joodi $i^{* *}$ \\ ${ }^{1}$ Surgical Oncology Research Center, Mashhad University of Medical Sciences, Mashhad, IR Iran \\ ${ }^{2}$ Department Radiology, Mashhad University of Medical Sciences, Mashhad, IR Iran \\ ${ }^{3}$ Faculty of Medicine, Mashhad University of Medical Sciences, Mashhad, IR Iran \\ "Corresponding author: Marjan Joodi, Surgical Oncology Research Center, Mashhad University of Medical Sciences, Mashhad, IR Iran. Tel: +98-9155031236, E-mail: \\ joodim@mums.ac.ir
}

Received 2017 December 19; Revised 2018 January 20; Accepted 2018 January 29

\begin{abstract}
Objectives: The present study aimed to investigate the efficacy of the ultrasound-guided wire localization of the anal tract and sphincter muscle complex in the patients with imperforate anus.

Methods: This study was conducted on 20 patients ( 4 females and 16 males) with imperforate anus referring to the Doctor Sheikh and Akbar Pediatric Hospitals, Mashhad, Iran, between2016 and 2017. The trans-perineal ultrasonography was performed under general anesthesia to identify the location and direction of the anal tract, which was checked by a muscle stimulator. Then, the localization needle was inserted in the center of the anal pit and anal sphincter muscle complex until it reached the rectal pouch. Eventually, by using the wire as a guide, the rectum was brought to the middle of the sphincter complex through the minimally invasive pull-through procedure.

Results: The anal sphincter muscle complex (concentric hypoechoic muscle) could be seen in all patients with the thickness of 1.8 $3.6 \mathrm{~mm}$ and mean of $2.6 \mathrm{~mm}$. The anal pit (the multi-layer view) was visualized in all patients, except in two cases with clinically lowtype imperforate anus. Muscle stimulator findings showed that the wire was located in the middle of the sphincter muscle complex in all patients. Mean localization time was $38 \mathrm{~min}$.

Conclusions: The ultra sound-guided wire localization of the anal tract facilitated the implementation of a less invasive and anatomically corrected rectal pull-through operation in patients with imperforate anus.
\end{abstract}

Keywords: Anal Sphincter Muscle Complex, Anorectal Malformation (ARM), Imperforate Anus (IA), Child

\section{Background}

Imperforations is one of the anorectal malformations characterized by abnormal development of anus and rectum. This defect is divided into high and low types. In the high type, the rectal end is above the sphincter muscle, while in the low type; the rectal end enters into the sphincter muscle complex and passes partially or completely through it (1).

The achievement of fecal continence is the most important goal in the surgical treatment of the patients with imperforate anus. This objective is accomplished by the diagnosis of the correct position of sphincter complex muscles followed by fixing an orectum to the sphincter complex (2). Various surgical procedures are applied to pull the rectum through the center of anal sphincter.

The posterior sagittal anorectoplasty (pull-through procedure) is the most common operation, which has the sufficient ability to improve the surgical visualization of the muscle complex (3). Although this method allows the accurate placement of the rectum, it requires a large incision and a complete division of the sphincter complex that probably hurts and weakens the muscle $(4,5)$.

The laparoscopic-assisted an orectoplasty is a new and more advanced surgical technique performed with or without the use of preoperative magnetic resonance imaging (MRI) for the diagnosis of the type and position of anorectal malformation. The limitation of this technique is its unreliable proper anatomic placement, which is due to the curved non-linear pathway of the sphincter muscles $(3,4,6,7)$.

Recently, fewstudies have investigated the use of MRIguided laparoscopic-assisted an orectoplasty for the detection of sphincter complex, an altract localization, and sur- 
gical guidance during the surgery (4-6). In this method, first, the sphincter complex is carefully mapped using an operative MRI suite. Then, with MRI guidance, a needle is precisely penetrated into the center of the sphincter complex. Ultimately, a less invasive laparoscopic operationis performed through the needle guide, and the rectumis pulled through the center of the sphincter complex without weakening the muscles that are required for continence (4-6).

Although this minimally invasive technique results in placing rectum through center of the sphincter, it has its own drawbacks, such as expensiveness and unavailability in all pediatric surgery centers. In our hospital, we used pre-surgical hook wire localization according to the ultrasound landmarks of the anal pit and sphincter muscle complex as operation guidance for rectal pull. The aim of this study was to present our experience in the application of ultrasonography images to guide a wire localization of the anal tract and sphincter muscle complex in the patients with imperforate anus.

\section{Methods}

This descriptive cross-sectional study was conducted on 20neonateswith imperforate anus ( 4 females and and 16 males) within the age range of 2 days to 12 weeks referred to the Doctor Sheikh and Akbar Pediatric Hospitals, Mashhad, Iran, during 2016 - 2017. The present study was approved and granted by the Institutional Review Board and ethic committee of the Mashhad University of Medical Sciences, Mashhad, Iran (Ir.mums.fm.rec.1386.131) and Thai Clinical Trials Registry (TCTR20171213001). Informed consent was obtained from the neonates' parents.

Out of the 20 participants, 13patientshad previous colostomy at the age of two days. Among the other seven neonates, one case had recto-vestibular fistula, and six subjects were suspicious of low types of imperforate anus. Under general anesthesia, the children were placed in lithotomy position, and Foley catheter was passed from the distal limb of colostomy into the rectum. The balloon was inflated, retracted backward, and then fixed.

For the better visualization of the rectal pouch, the distal pouch was filled with normal saline via the stoma. After prep and drape, the trans-perineal ultrasonography was performed by an expert pediatric radiologist. Sterile gel and betadine were applied for appropriate ultrasonic window. The ultrasound device used in this study was Sono Site Model S Nerve (Sono Site, Inc., USA) with a 12 MHz linear superficial probe.

At first, the exact sonographic examination of the perineal area was performed in sagittal and coronal planes to detect the position and state of the anal pit, sphincter complex, and internal fistula, location and direction of the anal tract through the middle of anal pit, and type of imperforate anus (Figure 1). The anal pit was detected as a multi-layered structure that was exactly similar to gut signature. The anal pit was only visualized in the coronal plane as a peripheral hypoechoic layer with two central parallel echogenic lines.

The anal sphincter complex was also visible in the coronal plane as a circular muscular tissue in the depth of the subcutaneous perineal area. Internal fistulas can be identified by changing the rout of the echogenic linear tract of the rectum toward urethra or vagina. The location and direction of the anal tract through the center of the anal pit and sphincter muscle complex were variable and different in terms of the shortest line between the rectal pouch and the surface of the skin. This path was mostly oblique and non-straight.

After the exact perception of perineal anatomy and anal route, the pediatric surgeon checked the proper location of the sphincter with a muscle stimulator. Then, in the coronal view, the needle of wire localization was inserted in the center of the anal pit. The localization was performed using the TSK breast localization needle (20 G, 100 $\mathrm{mm}$ ). The needle was propelled to the anal tract (i.e., the trace of anal pit center to the muscle sphincter center) with continuous switching in coronal and sagittal views, until it reached the rectal pouch (Figures 2 and 3 ).

If the proper localization of the needle in the middle of the anal tract, especially the center of the sphincter muscle complex, wasa chieved, the needle was withdrawn, and the hook of wire tip remained and was fixed in the rectal pouch, so the wire localization was complete. For better operational planning, the distance between the rectal pouch and the surface of the skin in the wire pathway (i.e., anal tract) and between the place of wire insertion inside the rectal pouch and the site of internal fistula were measured and documented in a sagittal view.

Subsequently, the pediatric surgeon rechecked the proper location of the wire with a muscle stimulator. After insuring the exact localization, the anal tract was precisely opened through the center of the sphincter complex with dilatators using the wire as a guide. Eventually, the rectum was placed through the center of the sphincter complex, and a less invasive pull-through operation was performed.

\section{Results}

Out of the 20 patients, 14 cases had high and 6 cases low type imperforate anus. The anal sphincter muscle complex was observed in all patients, although in two patients with recto vesical fistula, the muscle sphincter was not completely hypoechoic and its differentiation from is chiop- 

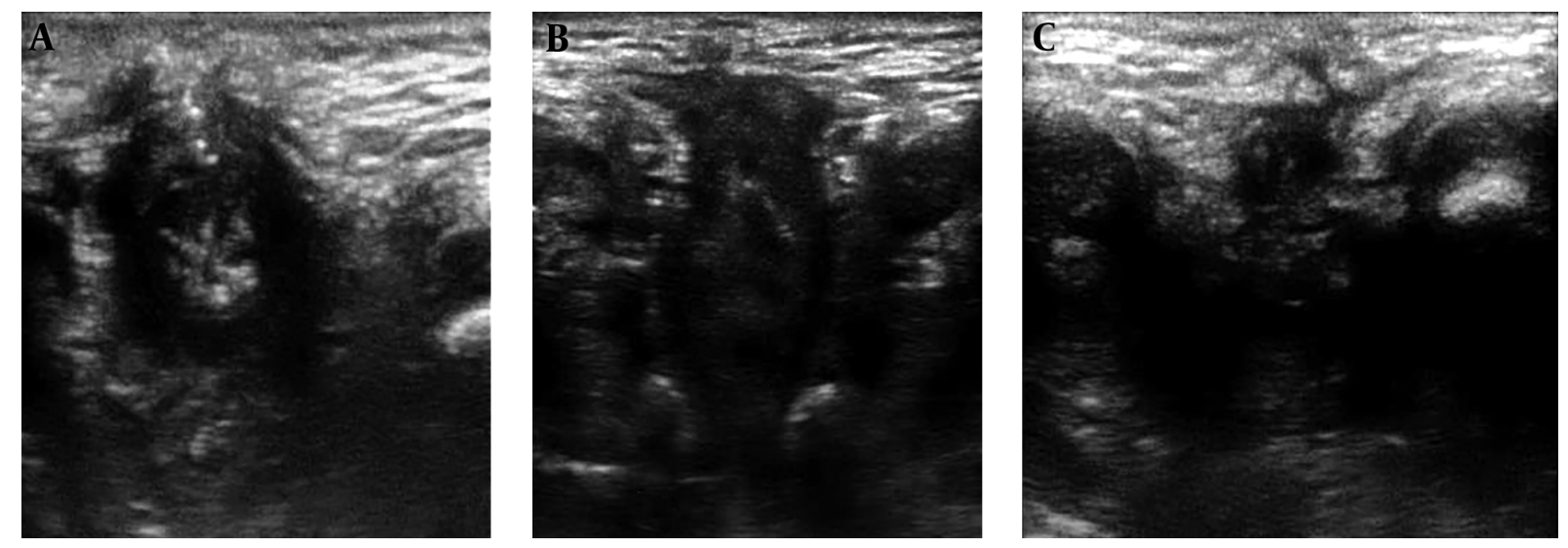

Figure 1. Coronal ultrasound images of anal tract in imperforate anus, A) a low type case: echogenic wrinkled mucus in the middle of hypoechoic muscular sphincter, B) another low type case: a part of rectum enters to the proximal part of sphincter complex, C) a high type case: eccentric anal pit and contracted muscle sphincter without visualization of rectal mucus
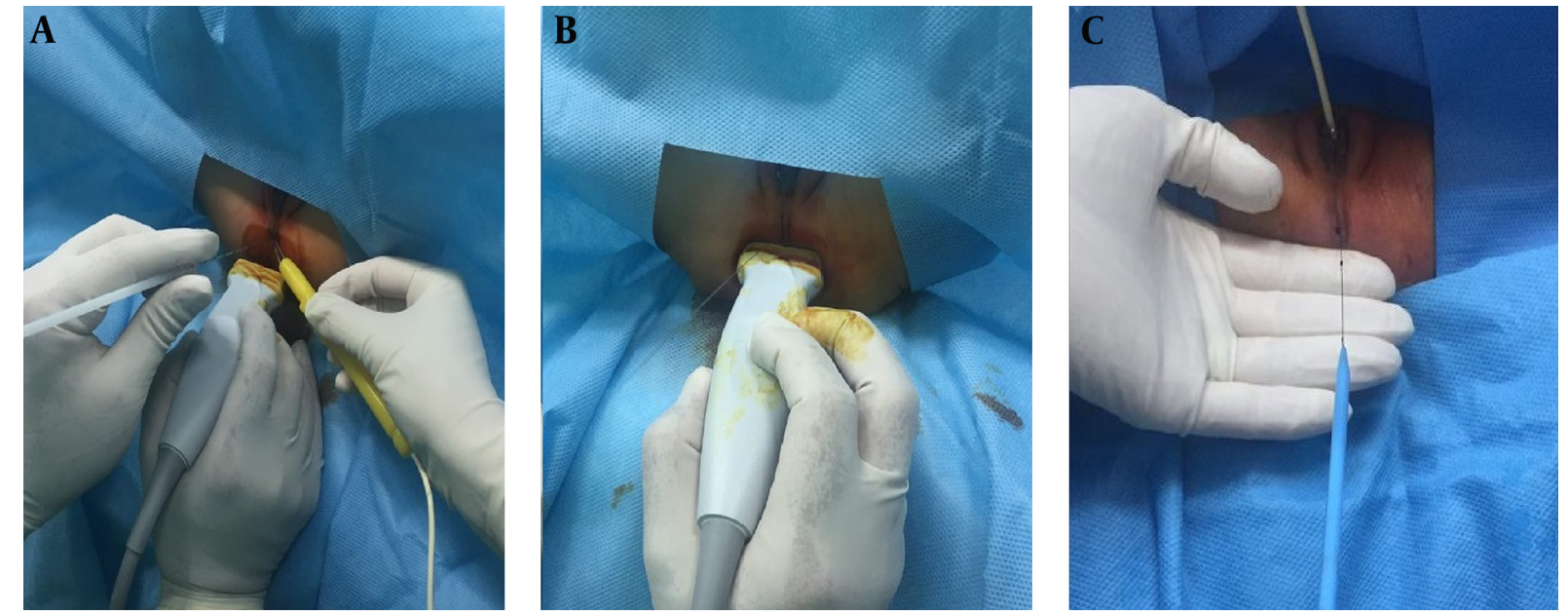

Figure 2. A, B) Technical images of ultrasound-guided localization of anal tract: usage of coronal and sagittal views for the anatomical detection of anal tract and application of muscle stimulator for matching anatomical and physiological anal complex muscle sphincter, C) final check of wire location in the center of anal tract

ubic fat was hard. Fiber alignment and usage of muscle stimulator we are completely helpful.The muscle complex thickness was 1.8-3.6 mm with a mean of $2.6 \mathrm{~mm}$, which was occasionally asymmetric. The anal pit was observed in all patients, except in two cases with clinically low type of imperforate anus and recto-vestibular or recto-perineal fistula. The alignment of the middle of the anal pit with the center of the sphincter muscle complex was eccentric or concentric; nevertheless, it was mostly no-straight and had a curved pathway.

The distance between the rectal pouch and the surface of the skin via the anal sphincter pathor the length of anal tract was within $11-28 \mathrm{~mm}$.

In all patients but two, anal pit had a concentric figure and the external coronal diameter of anal pit was $2.3-8 \mathrm{~mm}$ (with the maximum of $5 \mathrm{~mm}$ in high and $8 \mathrm{~mm}$ in low-type malformation). Ina female patient with cloacal anomaly and in one male patient with high type malformation, anal pit was of a band like configuration and its sagittal diameter was longer than coronal diameter, more than $10 \mathrm{~mm}$ on sagittal plan. Localization of anal pit in these patients with ultrasound alone was difficult and we had to use muscle stimulator. Only in one patient with low type malformation, anal pit was double in coronal plan. All patients had one type of fistula, except for two cases with Down syndrome.

In the patient with recto-vestibular fistula, the anal sphincter complex was pushed back toward the coccygeal 

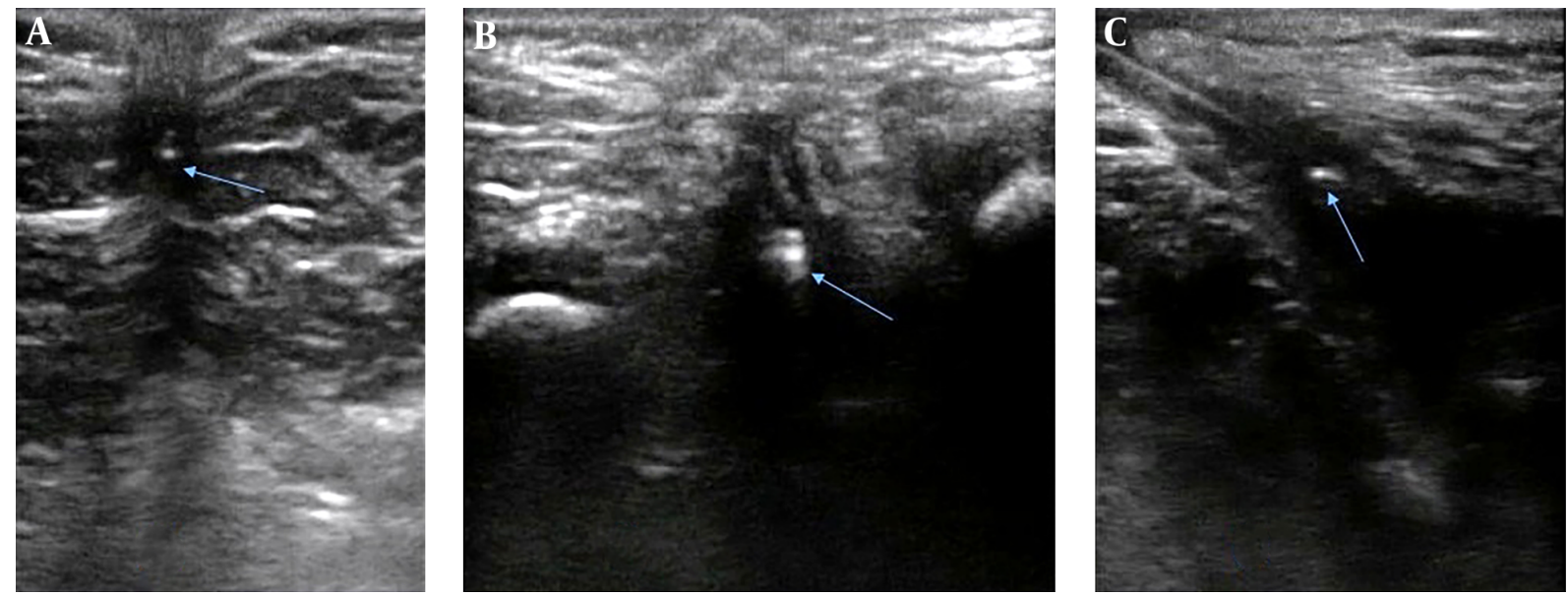

Figure 3. Coronal views of ultrasound-guided localization of anal tract, the arrows show A) echogenic tip of needle in anal pit and B) center of sphincter muscle complex, C) sagittal view of needle length in the middle of hypoechoic anal tract

tip probably due to fecal material pressure. Based on the intra-operation muscle stimulator findings, location of the wire was in the center of the sphincter muscle complex in all patients. The localization time was between 20 (inlow type) and 90 min (in high type) with the mean time of 38 min. The operation lasted 20 - 58 min (mean: $38 \mathrm{~min}$ ). In one patient, the wire localization was performed for asecond time toselect a better route.

\section{Discussion}

In imperforateanus patients, post-surgery fecal continence depends mostly on the exact identification of sphincter muscle complex and anal reconstruction. The reare some articles investigating the role of MRI in the determination of the type and level of anorectal malformation, which can be helpful in the pre-operative planning (1). The post-operative MRI aids in evaluating surgical outcome and the passage of pulled-through bowel from the center of the sphincter complex $(2,3)$.

Until now, the sonography was mainly used to indirectly determine the level of disorder based on the distance of the perineal skin surface to the rectal pouch and the existence of internal fistula (7-10). Our results demonstrated that the visualization of the anal sphincter complex and rectal pouch by sonography could facilitate the diagnosis of the exact level of malformation. The majority of our patients had high-type imperforate anus.

Reviewing the literature, we found only one study that investigated the differentiation of high type of this anomaly with its low type by the detection of the rectal passage from muscle with a different approach (11). We had two patients presented with constipation diagnosed with imperforate anus along with recto-vestibular and rectoperineal fistula in the clinical examination.

Conventionally, the patients with imperforate anus and recto-perineal fistula are categorized as low-type. In this patient, the distance of the rectal pouch to the skin was $9 \mathrm{~mm}$ based on the findings of the routine perineal ultrasonography, which categorized the patient in low-type malformation. However, further exact ultrasound examination in this patient revealed that she had a high-type malformation since the sphincter complex was pushed backward to the near of coccyx probably due to fecal retention.

The condition of this case revealed that the distance between the rectal pouch and skin without paying attention to the sphincter muscle was unreliable and could make a serious pitfall and complications. This case indicated the importance of preoperative imaging even in the clinically low-type defects as emphasized in some studies $(12,13)$.

Our findings demonstrated that while the ultrasound facilitated the visualization of the sphincter muscle complex in all patients, the anal pit visualization was possible in most of the patients. This visualization was helpful in the preoperative localization of the anal tract and the selection of less invasive surgical approach (i.e. pullthrough operation) resulting in better future fecal continence. Muscle stimulator findings were correlated with sonographic findings regarding the place of sphincter muscle.

In addition, according to the intra-operation muscle stimulator findings, the location of wire was in the center of the sphincter muscle complex in all patients. In the 
ultrasound-guided wire localization, the rectum passes accurately through the center of the sphincter complex without the incision of muscle tissues, which was performed in the conventional routine surgeries. This ultrasoundguided method is less time consuming than the MRIguided method (38min versus $96 \mathrm{~min}$ ) (5).

In the present study, the anal pit could not be visualized in two patients with superficial perineal fistula. Sagittal band-like configuration and double tract were other types of anal pit. Moreover, two patients with Down syndrome had no internal fistula. Regarding this and given the parasagittal position and eccentric alignment of the middle of the anal pit to the center of the muscle complex, it is essential to conduct more embryologic investigations that can explain the pathophysiology of this anomaly.

This technique has some priorities over the MRIguided methods, the most important of which are availability, simplicity for learning and practice, time- and costeffectiveness, as well as fixation of hook wire in the rectal pouch. Nevertheless, it should be noted that the anal pit and sphincter complex were more easily visible in the coronal view than in the routine sagittal view. This was a preliminary cross-sectional short time study and the patients will be followed up after surgery fora long time regarding complications and results in order to compare the outcome of this method with routine surgical approaches without ultrasound guide. The MRI and ultrasound correlational studies or postoperative evaluations with MRI may provide more reliable findings in this regard. Furthermore, performing studies entailing patient follow-up would demonstrate the functional efficacy of this therapeutic approach.

\section{Conclusions}

In the imperforate anus patients, given the multi-layer view of the anal pit and muscular bulk of anal sphincter complex, the ultrasound-guided wire localization of the anal tract helps to perform a less invasiveandan atomically correct rectal pull-through operation.

\section{Acknowledgments}

The present study was supported by the Research Deputy of the Mashhad University of Medical Sciences. The authors gratefully acknowledge the contribution of the members of the operation room of Doctor Sheikh and Akbar Hospitals for supporting us to conduct this study.

\section{References}

1. Tang ST, Wang Y, Mao YZ. MRI of anorectal malformations and relationship of the developmental state of the spincter muscle complex with fecal continence World. J Pediatr. 2006;3:223-30.

2. Wong KK, Khong PL, Lin SC, Lam WW, Lan LC, Tam PK. Postoperative magnetic resonance evaluation of children after laparoscopic anorectoplasty for imperforate anus. Int J Colorectal Dis. 2005;20(1):33-7. doi: 10.1007/s00384-004-0620-6. [PubMed: 15322835].

3. Eltomey MA, Donnelly LF, Emery KH, Levitt MA, Pena A. Postoperative pelvic MRI of anorectal malformations. AJR Am J Roentgenol. 2008;191(5):1469-76. doi:10.2214/AJR.07.3773. [PubMed: 18941087].

4. Raschbaum GR, Bleacher JC, Grattan-Smith JD, Jones RA. Magnetic resonance imaging-guided laparoscopic-assisted anorectoplasty for imperforate anus. J Pediatr Surg. 2010;45(1):220-3. doi: 10.1016/j.jpedsurg.2009.10.033. [PubMed: 20105607].

5. Thomas TT, Teitelbaum DH, Smith EA, Dillman JR, Vellody R, Jarboe MD. Magnetic resonance imaging (MRI)-assisted laparoscopic anorectoplasty for imperforate anus: a single center experience. Pediatr Surg Int. 2017;33(1):15-21. doi: 10.1007/s00383-016-3995-x. [PubMed: 27722897].

6. Thomas T, Teitelbaum DH, Vellody R, Spremo D, Elkins S, Ladouceur $\mathrm{R}$, et al. Development of an operative suspension system for the performance of MRI-OR-guided laparoscopic anoplasty. Pediatr Surg Int. 2016;32(3):301-6. doi: 10.1007/s00383-015-3838-1. [PubMed: 26602208].

7. Alehossein M, Mollaeian M, Salamati P. Validity of Transperineal Ultrasound in Infants with Imperforate Anus. Iran J Radiol. 2004;2(1-2):43-7.

8. Nemati M, Ataei OR, Aslanabadi S. Assessment of diagnostic value of transperinealultrasonography in determining type of imperforate anus. J Am Sci. 2013;9(10s):91-5.

9. Haber HP, Seitz G, Warmann SW, Fuchs J. Transperineal sonography for determination of the type of imperforate anus. AJR Am J Roentgenol. 2007;189(6):1525-9. doi: 10.2214/AJR.07.2468. [PubMed: 18029895].

10. Choi YH, Kim IO, Cheon JE, Kim WS, Yeon KM. Imperforate anus: determination of type using transperineal ultrasonography. Korean J Radiol. 2009;10(4):355-60. doi: 10.3348/kjr.2009.10.4.355. [PubMed: 19568463]. [PubMed Central: PMC2702044].

11. Han TI, Kim IO, Kim WS. Imperforate anus: US determination of the type with infracoccygeal approach. Radiology. 2003;228(1):226-9. doi: 10.1148/radiol.2281011900. [PubMed: 12738873].

12. Elsayed RF, Kamal HA, Liethy NE. Recent advances in MRI in the preoperative assessment of anorectal malformations, Egyptian. J Radiol Nucl Med. 2016;47:711-21.

13. Thomeer MG, Devos A, Lequin M, De Graaf N, Meeussen CJ, Meradji $\mathrm{M}$, et al. High resolution MRI for preoperative work-up of neonates with an anorectal malformation: a direct comparison with distal pressure colostography/fistulography. Eur Radiol. 2015;25(12):3472-9. doi: 10.1007/s00330-015-3786-0. [PubMed: 26002129]. [PubMed Central: PMC4636514]. 